\title{
Neurocognitive evidence of enhanced implicit temporal processing in video game players
}

Francois Foerster ( $\nabla$ francoisfoerster@gmail.com )

University of Strasbourg https://orcid.org/0000-0001-9454-4815

Matthieu Chidharom

Anne Bonnefond

Anne Giersch

Article

Keywords:

Posted Date: February 24th, 2022

DOI: https://doi.org/10.21203/rs.3.rs-1384616/v1

License: (c) (i) This work is licensed under a Creative Commons Attribution 4.0 International License.

Read Full License

Version of Record: A version of this preprint was published at Communications Biology on October 11th, 2022. See the published version at https://doi.org/10.1038/s42003-022-04033-0. 


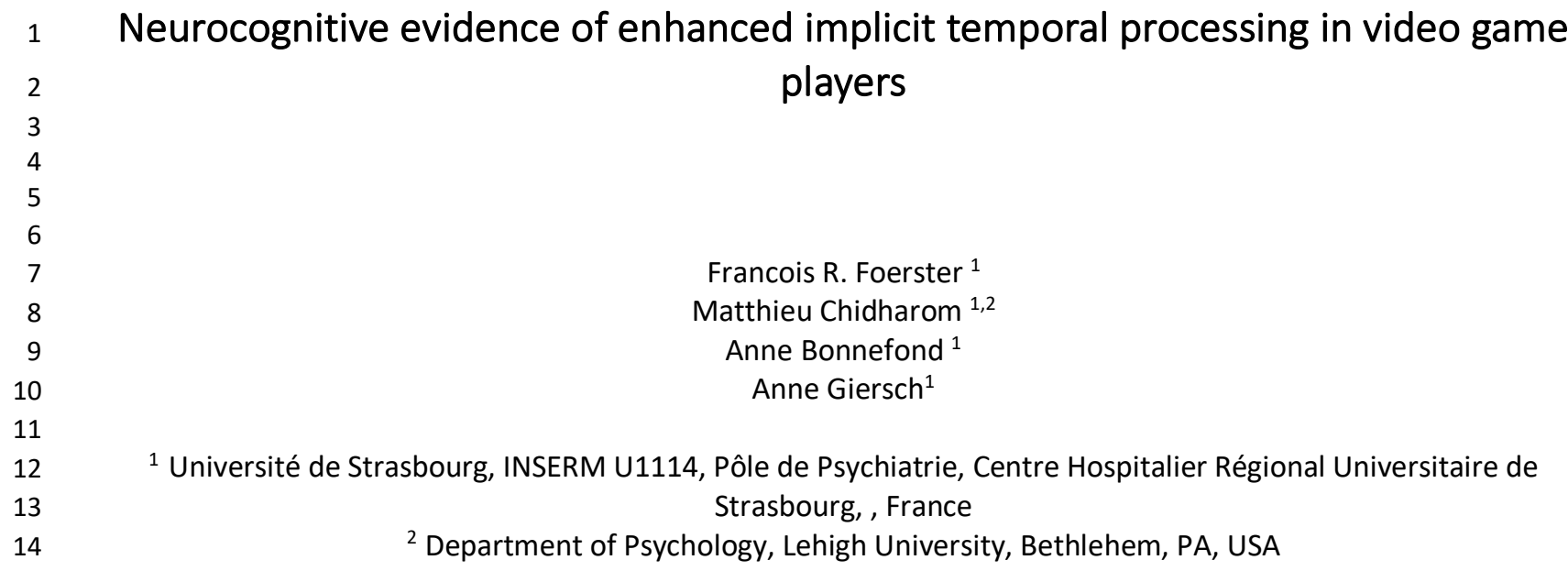

This work was supported by the European Union's Horizon 2020 FET program under the grant agreement [824128], VIRTUALTIMES consortium.

\footnotetext{
Francois Foerster, INSERM U1114, Pôle de Psychiatrie, Centre Hospitalier Régional Universitaire of Strasbourg, Université de Strasbourg, France

E-mail: francoisfoerster@gmail.com
} 
Abstract

Winning in action video games requires to predict timed events in order to react fast enough. In these games, the temporal structure of events is repetitive enough to develop implicit (automatic) preparation mechanisms. We compared action video game players (VGPs) and non-VGPs in a reaction time task involving both implicit time preparations and explicit (conscious) temporal attention cues. Participants were immersed in virtual reality and instructed to respond to a visual target appearing at variable delays after a warning signal (WS). In half of the trials, an explicit cue indicated when the target would occur after the WS. Behavioral, oculomotor and EEG data consistently indicate that, compared with NVGPs, VGPs better prepare in time using implicit mechanisms. This sheds light on the neglected role of implicit timing in VGPs and related electrophysiological mechanisms. The results further suggest that game-based interventions may help remediate timing alterations found in psychiatric populations.

Keywords:

Video games, temporal processing, saccades, phase-amplitude coupling, contingent negative variation 


\section{Introduction}

Video games are widely accessed and consumed at all ages. While playing video games can lead to internet gaming disorders ${ }^{1-3}$, within a few decades research showed that it can enhance cognition ${ }^{4-6}$. Game-induced cognitive enhancements depend on the type of game and their origins remain largely unknown ${ }^{7}$. In action video games, such as first-person shooter games, a key component is the ability to predict in time the appearance of visual targets, often foes, to prevail. In this case, being 'on time' involves both implicit and explicit time predictions ${ }^{8-11}$. Firstly, action video game play requires motor responses which are bound to include automatically timed preparation mechanisms ${ }^{12,13}$. Secondly, the temporal sequences of stimuli in these games are repeated from one match to another. Implicitly, players likely benefit from those repetitive sequences to refine expectations of forthcoming targets, which consequently might optimize their preparation and speed up their reactions. Such processing is considered implicit because players do not need to think about time. Therefore, the intensive training of action video game players might improve implicit (automatic) temporal mechanisms. In addition, players might use explicit temporal cues in games. For instance, they might explicitly use a visual cue to predict the exact moment of targets in their visual field, i.e. consciously orienting their attention in time ${ }^{8}$. A large number of studies revealed that playing action video games improves explicit attention mechanisms ${ }^{14-21}$. However, except for one investigation ${ }^{13}$, these studies explored spatial but not temporal aspects of attention. We need to know which type of mechanisms are enhanced through gaming. In addition video games have been proposed as a potential rehabilitation tool for psychiatric disorders ${ }^{22,23}$. Knowing how video game play shapes the brain and behaviors will help to adapt these tools to pathologies.

Here, we investigated implicit and explicit prediction and preparation in time, in action video game players (VGPs) and non-video game players (NVGPs) using a variable foreperiod task in a virtual environment. In this task, participants had to anticipate to speed up their responses to a visual target. Virtual reality helps to get close to ecological conditions, enables an optimal commitment to the task and allows the gaze to be tracked in the 3D space using the embedded eye-tracking system of the headset. In this study, we tested whether reaction time performance is enhanced in VGPs and relies on enhanced implicit processing of the passage of time (hypothesis 1), enhanced explicit orientation of attention in time (also called temporal orienting; hypothesis 2), or both (hypothesis 3). EEG and oculomotor activities were concomitantly recorded to evaluate the neurocognitive mechanisms responsible for these potential enhancements.

In our task, a target occurs at varying delays after an initial warning signal, hence the name of variable foreperiod (FP) task. Foreperiods were either $400 \mathrm{~ms}$ (short FP) or $1000 \mathrm{~ms}$ (long FP). The warning signal and target were embedded in robots, which created an environment closer to video games and more entertaining. Participants reacted to the target by pressing a button as fast as possible. The probability of target occurrence increases with the elapsing time and participants benefit from the passage of time to prepare their response, leading to faster reaction times in long than short FP ${ }^{24-28}$. This preparation indexes the implicit processing of the passage of time ("neutral cue condition"). In contrast, in temporal orienting a cue (in our case a robot's color, Figure 1; "temporal cue condition") explicitly indicates the foreperiod, so the participant knows the timing of the target occurrence. The cue orients attention in time and yields a decrease in reaction time.

Several neurobiological indexes are associated with temporal processing. The EEG contingent negative variation (CNV) is a neuronal signal known to be increased during temporal orienting 29-35, whereas theta-band oscillations have been found to increase when a visual target is implicitly expected ${ }^{36}$. Finally, temporal orienting has been associated with small fixational saccades, called microsaccades, which are inhibited before the onset of a temporally-predictable sensory signal ${ }^{37-40}$. Here, all evidence shows enhanced implicit temporal processing in VGPs.
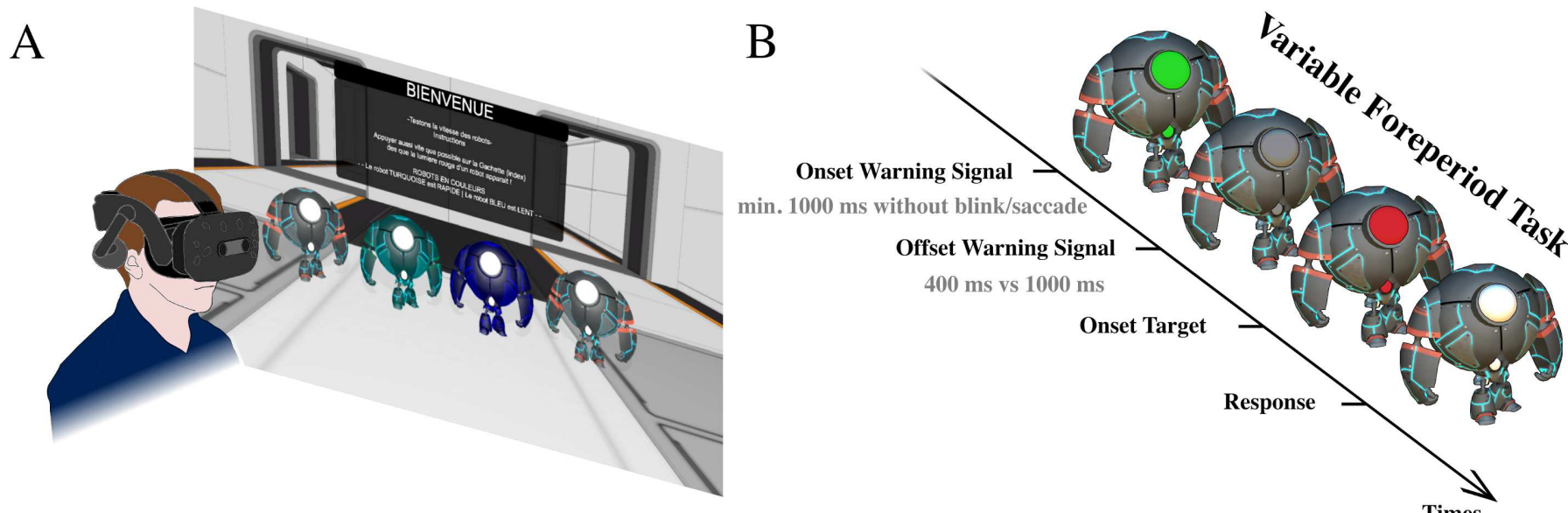

Figure 1. Virtual reality setup and experimental task. (A) Action video game players (VGPs, N=23) and non-video game players (NVGPs, $\mathrm{N}=23$ ) were immersed in a virtual environment with robots and performed a variable foreperiod task. (B) A short (400 $\mathrm{ms})$ or long $(1000 \mathrm{~ms})$ foreperiod separated in time the offset of an initial warning signal (i.e. a green light) and the onset of a target (i.e. a red light). The task of the participants was to press a button as fast as possible after the appearance of the target. 
The color of the robots served as a predictive cue (blue and turquoise robots; "temporal cue condition") or not (grey robots; "neutral cue condition") for the timing of the target. Reaction times, eye-tracking, and EEG measures were used to evaluate whether or not, and how, VGPs and NVGPs benefited from implicit temporal expectations and explicit temporal attention to optimize their performance.

\section{Results}

\section{Reaction times.}

VGPs are believed to be impulsive (but see ${ }^{41}$ ). A preliminary analysis on premature responses (anticipation errors - responding before the target appearance) revealed no evidence of different impulsivity in VGPs and NVGPs (see Supplemental Results). Then, a three-way rANOVA with the factors Group, Cue and Foreperiod was performed on reaction times (Figure 2). No effect of the Group was revealed $(p=.162)$. However, significant main effects of the Cue (Mean Temporal $=346 \mathrm{~ms}, \mathrm{Cl}_{\text {Temporal }}=11.1 \mathrm{~ms} ; \mathrm{Mean}_{\text {Neutral }}$ $\left.=355 \mathrm{~ms}, \mathrm{Cl}_{\text {Neutral }}=9.6 \mathrm{~ms} ; \mathrm{F}(1,44)=11.9, p=.001 ; \eta_{\mathrm{p}}^{2}=.016\right)$ and the Foreperiod $\left(\right.$ Mean $_{\text {ShortFP }}=359 \mathrm{~ms}, \mathrm{Cl}_{\text {shortFp }}=9.8 \mathrm{~ms}$; Mean $\left._{\text {LongFP }}=342 \mathrm{~ms}, \mathrm{Cl}_{\text {LongFP }}=10.7 \mathrm{~ms} ; \mathrm{F}(1,44)=70.8, p<.0001 ; \eta^{2} \mathrm{p}=.056\right)$ were revealed. These effects indicated that participants were faster to respond when the foreperiod was long rather than short (i.e. implicit processing) and when the timing of the target was predictable (i.e. explicit processing). The rANOVA revealed an interaction effect between the Cue and the Foreperiod $\left(F(1,44)=22.5, p<.0001 ; \eta^{2} p=.004\right)$. Planned comparisons showed that the effect of the Foreperiod was absent in the temporal cue condition $(p>.11)$. All these results replicate those in the literature, showing that they are preserved in the virtual environment. Finally, the analysis also revealed a triple interaction between the Cue, the Foreperiod and the Group ( $F(1$, $\left.44)=4.89, p=.032 ; \eta^{2}{ }_{p}=.0008\right)$, with an effect of the Foreperiod in the neutral cue condition in VGPs $(p=.003)$ but not in NVGPs $(p=.12)$. Consequently, only VGPs benefited from the passage of time when the target was not temporally cued - they were faster when the foreperiod was long rather than short. However, the lack of effect in NVGPs might have been due to a larger inter-individual variability in RTs in comparison with VGPs (see Figure S1). Alternatively, VGPs may have learned to optimize the task performance more rapidly than NVGPs ${ }^{42}$. These effects might have caused the group difference. To verify these possibilities, we propose a novel approach to estimate the subject-wise benefits from both the implicit passage of time and explicit temporal orienting, and verified how performance evolved across the four trial blocks of the experiment.

Novel estimates of the passage of time and temporal orienting effects. According to the literature, participants should respond faster when the foreperiod is long rather than short ( $R T_{\text {LongFP }}<R T_{\text {ShortFP }}$ ) and when the foreperiod is predicted by the cue ( $R T_{\text {Temporal }}$ $\left.<\mathrm{RT}_{\text {Neutral }}\right)$. These effects represent the benefit provided by the passage of time and by temporal orienting, respectively. To evaluate these benefits, we computed subject-wise indexes that consider the between-subject variability of response times.

First, we quantified how much participants implicitly benefited from the passage of time. We calculated the percentage of speed change given the formula: Speed change $(\%)=100 *\left(\mathrm{RT}_{\text {short FP }}-\mathrm{RT}_{\text {long FP }} / \mathrm{R} \mathrm{T}_{\text {short } \mathrm{FP}}\right)$. A one-sample t-tests analysis revealed that all participants, independently of their group, benefited from the passage of time in both the neutral (all $p<.0001$ ) and temporal (all $p<.0015$ ) cue conditions, confirming that our calculation helps to better evidence the benefits of the passage of time. We then performed a three-way rANOVA with the factors Cue, Block and Group to assess whether VGPs took better advantage of the passage of time than NVGPs. The rANOVA showed a main effect of the Cue $\left(F(1,44)=19.77, p<.0001 ; \eta^{2} p\right.$ $=.054)$ and an interaction effect between the Cue and the Group $\left(F(1,44)=4.7, p=.036 ; \eta^{2} p=.013\right)$. VGPs benefited from the passage of time significantly more in the neutral cue condition ( Mean $_{\text {Neutral }}=6.92 \%, \mathrm{Cl}_{\text {Neutral }}=1.86 \%$ ) than in the temporal cue condition $\left(\right.$ Mean $\left._{\text {Temporal }}=3.53 \%, \mathrm{Cl}_{\text {Temporal }}=1.98 \%, p=.0013\right)$. This effect was absent in NVGPs $\left(\right.$ Mean $_{\text {Neutral }}=4.69 \%, \mathrm{Cl}_{\text {Neutral }}=$ $1.35 \%$, Mean Temporal $\left.=3.53 \%, \mathrm{Cl}_{\text {Temporal }}=2.01 \%, p=.25\right)$. Crucially, planned comparisons showed that the benefit of the passage of time was greater in VGPs than in NVGPs in the neutral cue condition $(p=.013)$ but not with the temporal cue condition ( $p$ $=.995)$.

Second, we quantified how much participants explicitly benefited from the temporal cue to speed up their response time for each foreperiod. We calculated the percentage of speed change given the formula: Speed change $(\%)=100 *\left(R T_{\text {Neutral }}-\right.$ $\mathrm{RT}_{\text {Temporal }}$ / $\left.\mathrm{RT}_{\text {Neutral }}\right)$. To evaluate whether VGPs took better advantage of the temporal cue than NVGPs, a three-way rANOVA was performed on these values with the factors Foreperiod, Block and Group. The analysis revealed a main effect of the Foreperiod $\left(F(1,44)=20.19, p<.0001 ; \eta^{2} p=.029\right)$ and an interaction effect between the Foreperiod and the $\operatorname{Group}(F(1,44)=5.75, p=.021$; $\left.\eta_{p}^{2}=.009\right)$. VGPs benefited from the temporal cue at short FP $(p=.0002)$ but not at long FP $(p=.85)$, resulting in a significant difference in the effect of the temporal cue between the two foreperiods ( $p=.0007)$. NVGPs benefited from the temporal cue at both foreperiods (all $p<.044$ ), hence no difference in the effect of the temporal cue between the two foreperiods $(p=.53)$. A control analysis revealed similar observations when considering, in the calculation of the indexes, the sum of the two conditions as denominators (complementary results on the effect of the FP at trial $t_{-1}$ on the reaction time at trial $t$ can be found in Supplemental Results). 
A

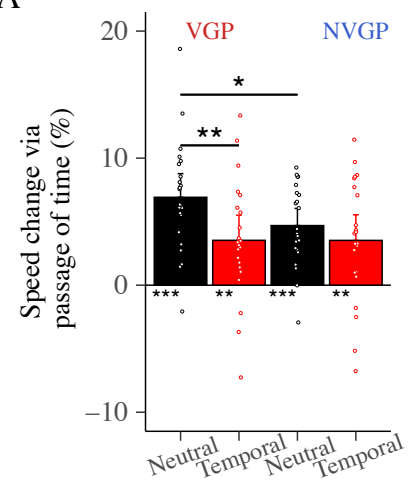

Cue
B

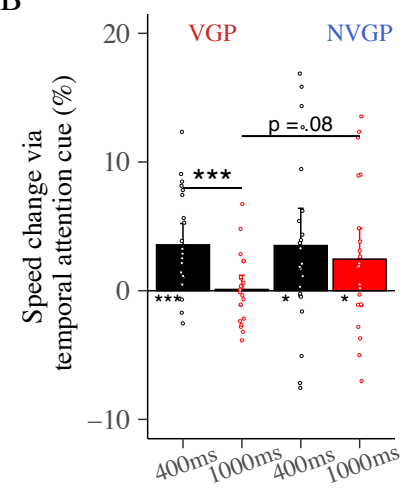

Foreperiod
A

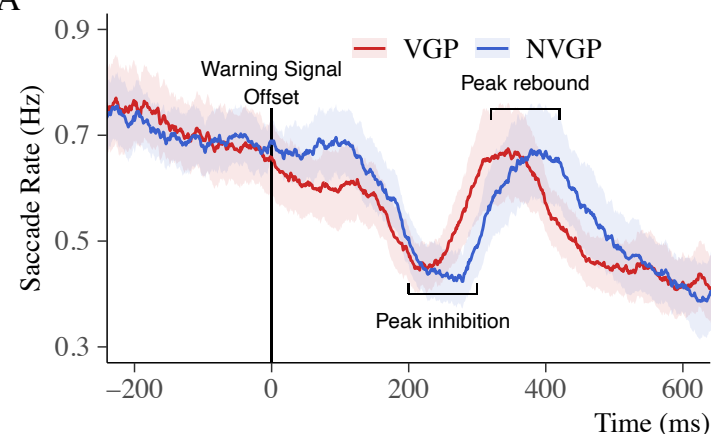

Figure 2. Behavioral results. Indexes were calculated to evaluate the effect of the implicit passage of time and explicit temporal attention on reaction times while accounting for inter-individual variability. To assess the benefit from the passage of time provided by a longer FP (A), an index was calculated as follows: speed change (\%) $=100$ * $\left(R T_{\text {short FP }}-R_{\text {long FP }} / R_{\text {short FP }}\right.$ ). Similarly, to evaluate the benefit from temporal attention provided by the temporal cue (B), another index was calculated as follows: speed change (\%) $=100 *\left(\mathrm{RT}_{\text {Neutral }}-\mathrm{RT}_{\text {Temporal }} / \mathrm{RT}_{\text {Neutral }}\right)$. Error bars represent \pm one confidence interval of the mean. ${ }^{*} \mathrm{p}<0.5, * * \mathrm{p}$ $<.01, * * * p<.001$. VGPs benefited more than NVGPs from the implicit passage of time.

Faster oculomotor responses in action video game players. We investigated the peak of reflexive saccadic inhibition and rebound 43-46 evoked by the offset of the initial warning signal representing the start of the waiting period. In other tasks, the saccadic inhibition represented the enhanced stimulus processing resulting from the top-down influence of attention 47,48 .

The latencies of the peak of saccadic inhibition and rebound are in accordance with previous studies that used more conventional eye-tracking systems ${ }^{44,47}$. To evaluate the oculomotor responses to the start of the waiting period, a three-way rANOVA with the factors Group, Foreperiod and Cue was conducted on both the latencies of the peak of inhibition and the latencies of the peak rebound extracted within the 100-300 ms and 300-500 ms time-windows, respectively. The analysis of the peak of inhibition revealed a main effect of the Group $\left(F(1,44)=4.7, p=.035 ; \eta^{2} p=.028\right)$ and indicated that the peak occurred significantly earlier in VGPs (Mean $=208 \mathrm{~ms}, \mathrm{Cl}=12 \mathrm{~ms}$ ) than in NVGPs (Mean $=226 \mathrm{~ms}, \mathrm{Cl}=11 \mathrm{~ms}$ ). No other effect was found (all $F(1,44)<1.4$, all $p>$.243). Similarly, the peak of saccadic rebound occurred earlier in VGPs relative to NVGPs (see Supplemental Results). Overall, the analysis suggests a faster oculomotor response in VGPs (Figure 3). Interestingly, a Pearson correlation revealed a significant negative correlation between the latency of the peak inhibition and the benefit from the passage of time ( $r$ $=-.33, p=.024)$, independently of the cueing condition and the group. This suggests that the benefit from the passage of time was more important in participants with short latency of peak inhibition (Figure 3C), which is particularly the case in VGPs. Finally, the temporal orienting phenomenon has been associated with small saccades during gaze fixation, which is inhibited before the onset of a temporally-predictable target ${ }^{37-40}$. We replicated such results with our paradigm (see in Supplemental Results).
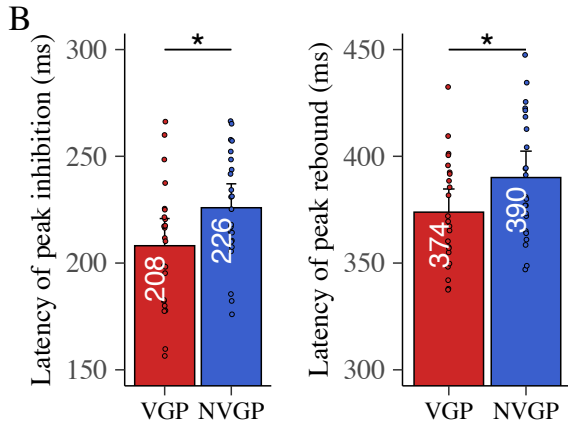

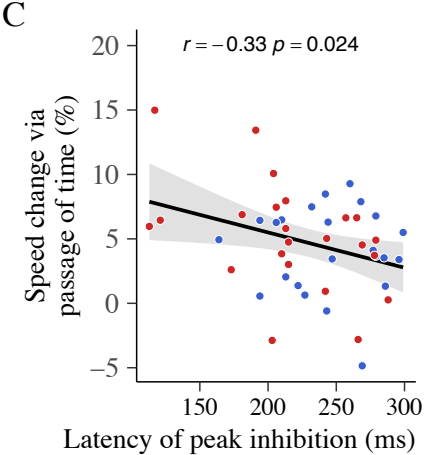

Figure 3. Dynamics of saccade rates. We observe an oculomotor reflex when the foreperiod starts (A). This oculomotor reflex was faster in VGPs (B). The negative correlation (C) between the benefit from the implicit passage of time and the latency of peak inhibition shows that participants' benefitting from the implicit passage of time had faster oculomotor reflexes. * $p<0.5$. Colored shaded areas represent \pm one SEM. Error bars represent \pm one confidence interval of the mean. 
CNV and temporal orienting. Several neurobiological markers are associated with temporal expectations. The contingent negative variation (CNV) is an EEG signal whose magnitude is increased during temporal orienting 29-35. A two-way rANOVA with the factors Group and Cue was conducted on the magnitude of the centro-parietal CNV (Figure 4) recorded within the $400-1000$ ms time interval in trials with long FP. This time interval starts at the time of the earliest possible occurrence of the target and ends when the target appears. The analysis did not report a significant effect of the Group $\left(F(1,43)=3.4, p=.071 ; \eta^{2} p=.062\right)$, but revealed a main effect of the Cue $\left(F(1,43)=7.2, p=.01 ; \eta_{p}^{2}=.029\right.$; Figure 4B-C; Figure S5) indicating that the magnitude of the $\mathrm{CNV}$ was larger in the neutral cue condition (Mean $=-5.48, \mathrm{Cl}=1.35$ ) than in the temporal cue condition $(\mathrm{Mean}=-3.99, \mathrm{Cl}=$ 1.33 , see Figure $4 D)$. No interaction effect $\left(F(1,43)=0.03, p=.88 ; \eta^{2}{ }_{p}<.001\right)$ was revealed. Hence, at first sight this result seems to contradict the literature.

Previous studies showed that the CNV slope is adjusted according to the temporal expectations, in a way that the magnitude of the CNV reaches its maximum around the expected time of a target appearance $30,31,37$. We evaluated the slope in trials with the long FP only, calculated as the difference between the averaged CNV in the 900-1000 ms time-interval and the averaged CNV in the 300-400 ms time-interval (at which time there was no target, since we considered trials with the long FP only), divided by the time difference between the two windows (i.e., $0.6 \mathrm{sec}$ ). Here, a flat or positive slope would indicate that the CNV peaked (i.e. was more negative) around the short FP, whereas a negative slope would suggest that the CNV peaked around the long FP. One-sample t-tests indicated the presence of a negative slope across all cues and groups $($ all $t(21,22)>2.24$, all $p<.036$, Figure $4 \mathrm{E})$ except in the temporal cue condition in VGPs $(\mathrm{t}(21)=0.93, p=.36)$. According to the literature, this result suggests that VGPs were equally prepared at short and long delays when they knew that the target would appear at 1000 ms. The Group or the Cue did not affect the steepness of the slopes (all $F(1,43)<1.91$, all $p>.175$ ). However, a Pearson correlation analysis revealed that participants who benefited from the temporal cue in trials with long FP had a more negative CNV slope in trials with the temporal cue rather than with the neutral cue $(r=-.323, p=.031$, see Figure $4 \mathrm{~F})$. This result supports the literature suggesting that the CNV slope reflects the explicit temporal orienting phenomenon ${ }^{37}$.

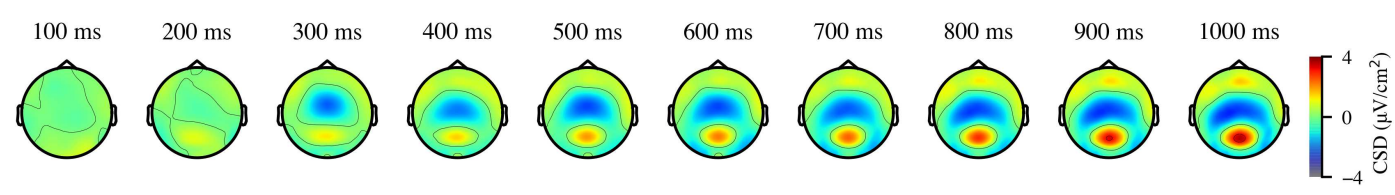

B

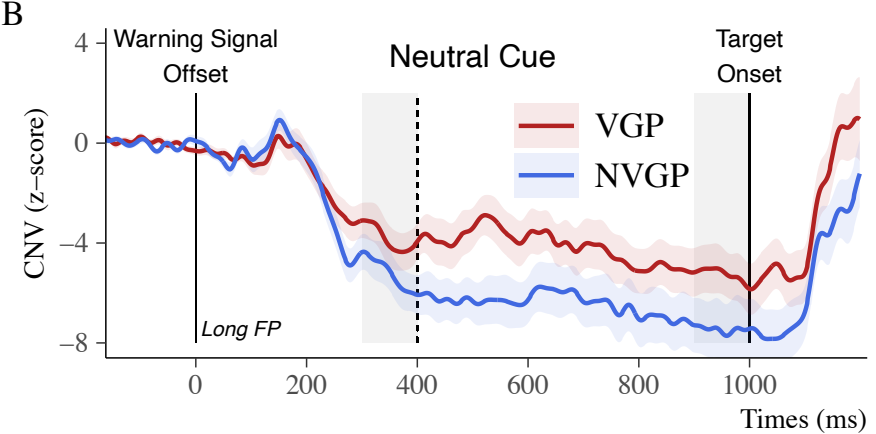

$\mathrm{D}$

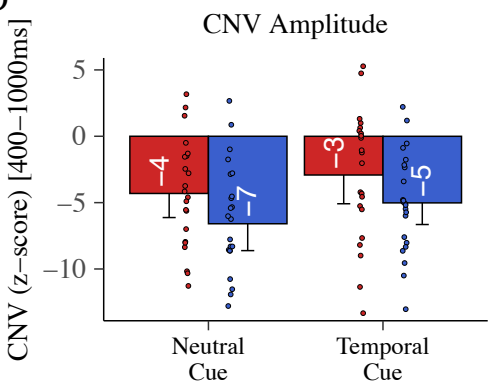

E

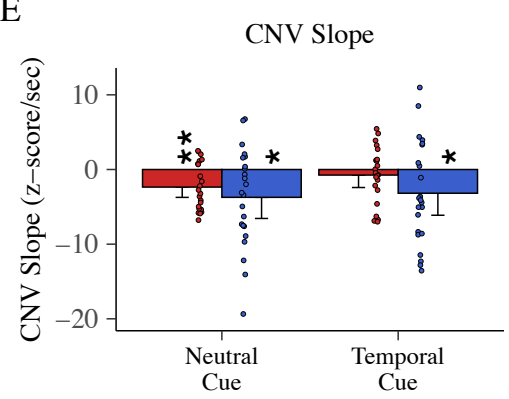

C

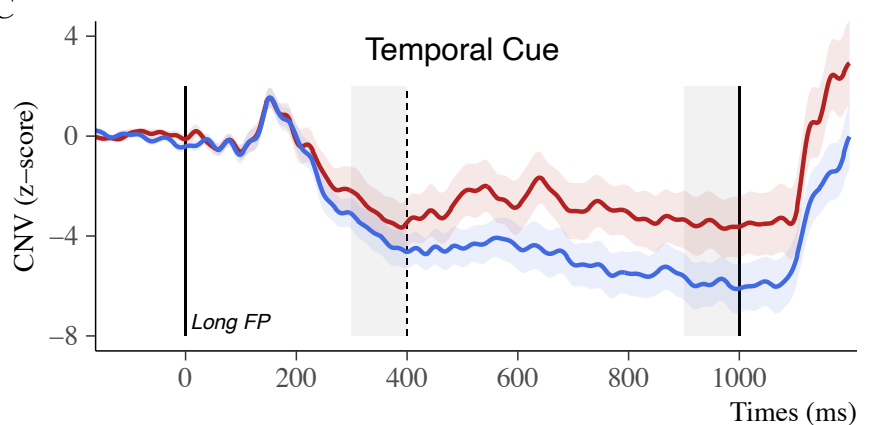

Figure 4. Contingent Negative Variation. Temporo-spatial evolution of the normalized CNV in trials with the long foreperiod across cueing conditions (A). In this analysis, we evaluated the explicit temporal orienting given 1) the averaged amplitude of the CNV within the 400-1000 ms time interval and 2) the slope of the CNV representing the amplitude difference between $1000 \mathrm{~ms}$ and $400 \mathrm{~ms}$. In trials with the neutral (B) and temporal cues (C), the amplitude of the CNV was not significantly reduced in VGPs relative to NVGPs. Grey areas represent the time intervals used to calculate the slope of the CNV. Independently of the group, 
the amplitude of the CNV was reduced in the temporal cue condition relative to the neutral cue condition (D). All CNV slopes were negative, except in VGPs in the temporal cue condition (E). Correlation analysis indicated that participants benefiting from the temporal cue in trials with the long FP exhibited a more negative CNV slope in the temporal rather than in the neutral cue condition (F). Colored shaded areas represent \pm one SEM. Error bars represent \pm one confidence interval of the mean. ${ }^{*} p<0.5$, ** $p<.01$.

Reduced theta oscillations in action video game players. In the literature, temporal expectations were reflected in the centrally recorded theta-band power and the centro-motor theta and beta phase-amplitude coupling (PAC) ${ }^{36}$. A two-way rANOVA with the factors Group and Cue was conducted on the averaged theta-band power in the 300-500 ms time interval (in the same time interval as in ${ }^{36}$ ), for trials with a long FP (Figure 5A-C). This time interval of 300-500 ms corresponds to the short foreperiod delay. During this time interval, the probability of target occurrence is $50 \%$ and $0 \%$ in the neutral and temporal cue conditions, respectively. Hence, at $300-500 \mathrm{~ms}$ in trials with a long foreperiod, target expectation is stronger in the neutral than in the temporal cue condition. The analysis reported a main effect of the Cue $\left(F(1,43)=6.05, p=.018 ; \eta^{2} p=.035\right)$, revealing that the magnitude of theta oscillations was increased in the neutral (Mean $=12.10, \mathrm{Cl}=2.65$ ) relative to the temporal cue condition (Mean $=8.86, \mathrm{Cl}=2.88$ ). Also, the analysis revealed a main effect of the $\operatorname{Group}\left(F(1,43)=6.49, p=.014 ; \eta^{2} p=.101\right)$, with a reduced magnitude of the theta oscillations in VGPs (Mean $=7.53, \mathrm{Cl}=2.08$ ) compared with NVGPs (Mean $=13.31, \mathrm{Cl}=3.11, \mathrm{Figure} 5 \mathrm{D}$ ). No interaction effect was reported $(F(1,43)=1.07, p=.307)$. These results suggest that 1$)$ theta-band activity was increased when the target probability occurrence was higher (i.e. in the neutral cue condition), thus supporting the link between temporal expectation and mid-frontal theta-band activities, and 2) VGPs had decreased temporal expectations relative to NVGPs when the probability of target occurrence was indeed low (i.e. 50 or $0 \%$ ).

Increased phase-amplitude coupling (PAC) in action video game players. The interplay of multiple brain rhythms permits efficient communication between distant cortical areas. To assess this communication, comodulograms were calculated on the 0-1000 ms time interval for trials with a long FP. Their visual inspection revealed a coupling between the phase of the frontocentral theta oscillations and the amplitude of the left motor beta oscillations (20 to $40 \mathrm{~Hz}$ range; Figure 6). Given the nonnormality of the $\theta-\beta$ PAC values (Shapiro-Wilk tests $p<.001$ ), non-parametric two-sided Wilcoxon signed-rank tests were used to evaluate significant differences across groups and cueing conditions. The PAC was evident in all groups and cueing conditions (all $p<.0001$; one-sample Wilcoxon signed-rank tests). The analysis revealed no main effect of the Group $(p=.478)$ or the Cue $(p$ $=.087)$. The $\theta-\beta$ PAC values were not statistically different between groups in trials with the neutral $(p=.067)$ or temporal $(p$ $=.71$ ) cue conditions. However, in VGPs these $\theta-\beta$ PAC values were significantly higher in the neutral cue condition (Mean $=$ $9.3 \times 10^{-5}, \mathrm{Cl}=3.9 \times 10^{-5}$ ) relative to the temporal cue condition (Mean $=6.8 \times 10^{-5}, \mathrm{Cl}=4.2 \times 10^{-5} ; p=.039$, "moderate" size effect $r$ $=.31$ ). This effect was absent in NVGPs $(p=.71)$. A Spearman correlation indicated a strong relationship between the logtransformed $\theta-\beta$ PAC values and the benefit from the implicit passage of time in the neutral cue condition (all $p<.013$, Figure 6D) but not in the temporal cue condition (Figure $6 \mathrm{E}$ ). These results expand previous findings ${ }^{36,49,50}$ but also suggest that participants benefiting from the implicit passage of time exhibit an increased fronto-motor functional oscillatory connectivity, which is especially the case in VGPs. Multiple control analyses strongly support the specificity of the $\theta-\beta$ PAC in the frequency-and spacedomains (see in Supplemental Results). 
A

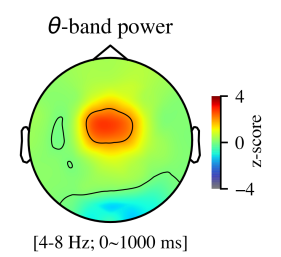

B

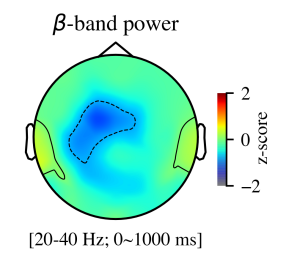

C

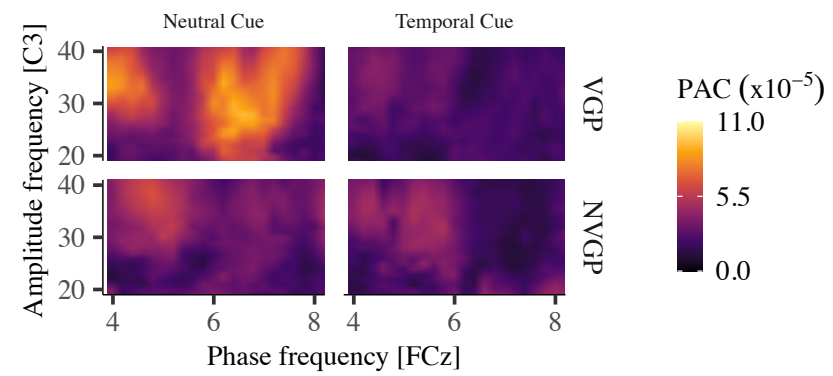

D

E
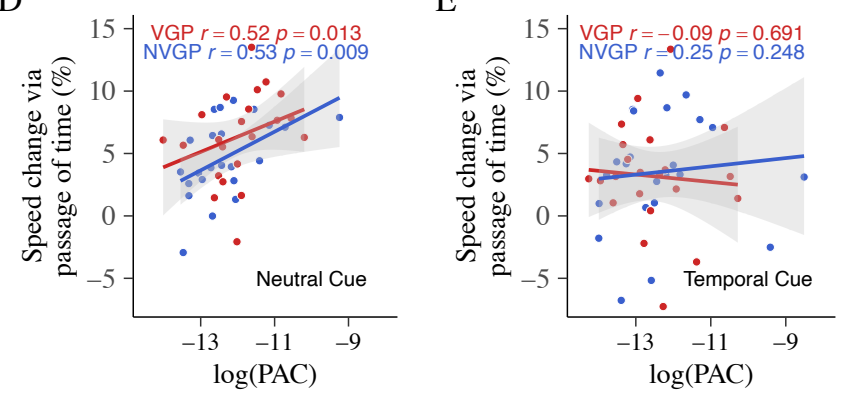

Figure 6. Fronto-motor phase-amplitude coupling (PAC). Oscillations in theta (A) and beta (B) frequency bands were localized around fronto-central and left motor areas, respectively. Comodulograms revealed a stronger $\theta-\beta$ PAC in the neutral cue condition than in the temporal cue condition (C). This effect was specific to the VGPs. A Spearman correlation (D) revealed a positive relationship between the participants' $\theta-\beta$ PAC (log-transformed values) and their benefit from the implicit passage of time (all participants). The correlation was significant with trials from the neutral cue condition (D) but not with trials from the temporal cue condition (E).

\section{Discussion}

In this study, both implicit processing of the passage of time and explicit temporal attention were investigated in VGPs and NVGPs using a visual variable foreperiod task. Two foreperiods defined two possible time intervals separating the offset of an initial warning signal (i.e. a green light) and the onset of a target (i.e. a red light). Small fixation saccades and EEG of participants were monitored while they were instructed to provide a fast-manual response to the target. In this paradigm, the timing of the target appearance was predictable based on 1) the implicit passage of time given the conditional probability that the target has not appeared yet and 2) the explicit temporal orienting given the attentional cue. The decrease of reaction times when the foreperiod was long rather than short indicated that both VGPS and NVGPs anticipated in time the target. Regarding our hypotheses, implicit rather than explicit temporal skills were improved in VGPs, such as VGPs benefited more from the implicit passage of time than NVGPs, a benefit most likely resulting from action video game practice. We found three mechanisms related to this implicit passage of time benefit. First, the reflexive saccadic response to the offset of the warning signal was enhanced in VGPs. This saccadic response predicted how much participants benefited from the passage of time, hence suggesting a relationship between the detection of the onset of a time interval of interest and the ability to track the elapsing time within this interval. Second, the midline frontal theta-band activity was reduced in VGPs when the probability of occurrence of the target was low, suggesting an adequate adaptation of expectation to the probability of target occurrence. Third, the EEG analysis revealed a fronto-motor phase-amplitude coupling during the foreperiod, supporting previous interpretations of this coupling as a mechanism of implicit temporal processing ${ }^{36}$. Separate analyses in each group confirmed an increased fronto-motor phaseamplitude coupling in VGPs while performing the task in the neutral cue condition. Overall, the results suggest that VGPs have optimized implicit temporal skills allowing them to deploy and withhold cognitive resources when suitable. 
The evidence illustrates enhanced automatic mechanisms in video game players allowing them to time their perception even when they do not have to think about time itself. Indeed, the goal of the participant was to react to the target and not to time the foreperiods. Enhanced implicit mechanisms in VGPs start with faster saccadic reflexes, indicating an improved processing of the offset of the warning signal. The correlation linking reflexive oculomotor response to (at least some aspect of) the processing of the elapsing time appears in line with the hypothesis that saccades modulate accumulation processes in the brain 51. In our task, tracking the elapsing time can be understood as a continuous accumulation of sensory evidence up to the target appearance. We speculate that faster saccadic reflexes permit to free cognitive resources, which in turn allows for more efficient accumulation processes ${ }^{51}$. Next, the theta-band analysis revealed that temporal expectations were lower in VGPs than in NVGPS when the probability occurrence of the target was indeed low (50 or $0 \%$ chance). This suggests that, generally, temporal expectations were better adapted in VGPs. This better adaptation could have allowed VGPs to deploy distinct mechanisms to perform the task in our two conditions. It would explain the stronger $\theta-\beta$ functional coupling in VGPs when dealing with the uninformative neutral cue relative to the temporal cue. This coupling could represent a sensorimotor updating mechanism 52 integrating the elapsing time to refine implicit expectations about the timing of the target. All things considered, implicit rather than explicit mechanisms appear optimized in VGPs.

In this variable foreperiod task, it is in the neutral cue condition that VGPs differed from NVGPs the most (hypothesis 1). It is not surprising given the importance of implicit temporal expectations to speed up responses in action video games, where the temporal structure of events is often predictable. Given the diverse evidence of improved attention in VGPs, enhanced benefit from the temporal cue might have been expected in these participants (hypothesis 2) but no proof was unveiled. Nevertheless, our data questions the EEG marker of temporal attention, namely the CNV. While we found increased CNV amplitudes when the timing of the target was neutrally cued, previous studies have reported increased CNV amplitudes when the timing of the target was temporally cued 33,34 . Crucially, in these studies the processing of the temporal cue was concomitant to the start of the waiting period, meaning an overlap of the encoding and usage of the temporal information and the continuous processing of the elapsing time. The paradigm presented here allows disentangling these two cognitive processes in assessing specifically whether and how the encoded temporal information helps to orient attention in time. With this important methodological distinction in mind, the pattern of CNV activity suggests that temporal attention reduces the neural cost of motor preparation, at least once the cued information has been processed. This result is consistent with fMRI data showing that temporal cue involves less activation in the right inferior frontal gyrus than neutral cue at long foreperiods, i.e. when updating is required ${ }^{53}$.

Current theories suggest that action video game play increases neural plasticity ${ }^{5}$, which in turn facilitates the rapid learning of the critical aspect of the task at hand ${ }^{54}$ and explains the perceptual and cognitive enhancements found in VGPs. Similarly, we believe that the benefit from the passage of time found in VGPs may relate to transfer learning mechanisms ${ }^{55}$. Such transfer learning mechanisms explain why playing specific video games can speed up phonological decoding ${ }^{17}$, enhance reading 56 and multitasking abilities ${ }^{16}$. Here we propose that being trained to orient the attention accurately in time helps implicit time expectations in general. We deem that further work should evaluate the causal effect of action video game play on implicit temporal processing, keeping in mind that game-based interventions could represent an affordable and engaging remediation tool for time perception alterations in psychiatric populations ${ }^{22}$.

\section{Method}

Participants. The VGP group concerned 23 participants ( 4 females, 2 left-handed, age Mean = 25.2; SD = 5.7). The criterion to be considered a VGP was a minimum of 5 hours per week of action video game practice for the previous 12 months, as reported in previous studies $18,21,54$. The games mainly included first-person shooters (e.g. Call of Duty series, Apex Legends, Overwatch, Counter Striker series), multiplayer online battle arena (e.g. Leagues of Legends, Heroes of the Storm), and real-time strategy (Starcraft II) which involve important visual and timing expectations, as well as high-speed visual processing and motor responses to optimize game performance. The NVGP group concerned 23 participants ( 7 females, 5 left-handed, age Mean = 26.8; SD = 4.6). The criterion to be included in the NVGP group was little or no action video game practice for a minimum of one year, although no extensive practice ever $(\mathrm{N}=16)$ was highly favored. All subjects had normal or corrected-to-normal visual acuity, as checked with the Freiburg Visual Acuity Test ${ }^{57}$. One VGP has been removed from the EEG analysis due to excessive noise in the recorded signal. All participants were given a compensation of $45 €$ for their participation and provided a written informed consent to take part in the study. The study has been approved by the local ethics committee of the University of Strasbourg (i.e. Comité d'Éthique de Recherche).

Experimental Protocol. The experiment used the Unity software (Unity technologies, v. 2019.3.9f1) to create the virtual environment. The HTC Vive Eye Pro (HTC Corp.) headset and controllers were used to immerse the participants in VR. Participants wore both the EEG and VR headsets while sitting on a chair. The use of VR has several advantages. Firstly, VR allows a better trade-off between fully-controlled experimental settings and ecological experience (e.g. 3D visual percepts) in comparison with 2D screen setups. Secondly, it increases the engagement of the participant to the task at hand. Thirdly, the embedded eyetracking system to the VR headset allows researchers to easily track and record the gaze in the 3D space. Eye-tracking was used to trigger visual stimuli. Participants were instructed to fixate the warning signal without moving their eyes, and it was only after 
a time interval free of saccades and eye-blinks that the warning signal was switched off. This procedure improves the data quality of EEG recordings.

Each participant was immersed in a virtual room, facing four 3D robots, each with a light whose color and onset were manipulated (Figure 1A). The experiment was composed of two intertwined tasks: a variable foreperiod task and an asynchrony detection task. The asynchrony detection task consisted of discriminating whether the lights of two robots appeared synchronously or asynchronously (using delays of $11 \mathrm{~ms}, 33 \mathrm{~ms}$, or $66 \mathrm{~ms}$ ). Participants performed four blocks of trials for each task. The participant switched tasks every block to reduce boredom. At the end of each experimental block, a break was proposed to the participant to remove the VR headset. Given the research questions investigated in this article, only data collected from the variable foreperiod task are presented.

Variable foreperiod task and stimuli. In case the button was pressed before the go-signal, a warning sound was delivered to the participant signaling the incorrect response. The procedure consisted of 4 blocks of 120 trials, with each block comprising 60 trials with short (S) FP and 60 trials with (L) long FP. The procedure excluded the possibility of having three same foreperiods in a row (i.e. SSS or LLL). The light of the robots was presented at a distance of 4 meters from the participant. These lights were located at $8^{\circ}$ and $24^{\circ}$ of visual angle from the center of the scene. The presentation of the temporal $(\mathrm{T})$ and neutral $(\mathrm{N})$ cue conditions was alternated, taking the form of NTNT $(N=24)$ or TNTN $(N=22)$. At the beginning of each block, the two robots used in the condition were relocated to the center of the scene and the two other robots were relocated on the sides (randomly on the right and left). At the beginning of each trial, a colored (during temporal cue blocks) or uncolored (during neutral cue blocks) robot was randomly selected to include the warning signal and the target light. The matching of the robot's color with the FP was randomly assigned for each participant: blue for the short FP and turquoise for the long FP, or the reverse.

Behavioral analyses. On the one hand, pressing the button before the onset of the target (i.e. anticipated responses) reveal the impulsivity ${ }^{58}$ in the two groups. On the other hand, pressing the button after the onset of the target was used to compute the two indexes (i.e. benefits from the passage of time and temporal attention cue).

Eye-tracking acquisition and analyses. The binocular gaze position was monitored using the eye-tracking system (Tobii Ltd.) embedded in the VR headset at a sampling rate of $90 \mathrm{~Hz}$ and an estimated spatial accuracy between $0.5^{\circ}$ to $1.1^{\circ}$. The particularity of such a system is that 1 ) it tracks the gaze position independently of head movements, 2) it provides estimations of the gaze location in the 3D space rather than on-screen 2D space, 3) the calibration-free data recording for saccades analysis renders the measure non-intrusive. Here we analyzed the likelihood of small fixational saccades during the foreperiods, as previously investigated $37,39,59$. Saccades of all sizes were included, but due to the task requirements to fixate the stimulus area, most saccades were small ( $1.4^{\circ}$ of visual angle on average).

First, the onsets of blinks were identified with the HTC SRanipal SDK, detecting blinks via individual eye openness. Because blinks were particularly rare events given to non-blinking requirements to trigger the offset of the warning signal, trials containing at least one blink occurring during the time-window of interest (i.e. -200 to $600 \mathrm{~ms}$ in trials with short FP; -200 to 1200 $\mathrm{ms}$ in trials with long FP) were discarded (5.3\% of total trials). Trials with anticipated responses were also discarded ( $1.85 \%$ of the data). Second, raw data (i.e. the 3D gaze position over time) of each trial were interpolated with a spline method to increase the temporal precision followed by the calculations of the derivations of the speed of vertical and horizontal movements.

Saccades were detected using a modification of a published algorithm ${ }^{60}$ based on gaze's velocities. A threshold criterion for saccades detection was determined in the 2D velocity space based on the horizontal and the vertical velocities of gaze movement. This 2D space represented a plane surface located at the stimulus area (i.e. the warning signal and target). This threshold was represented by a 2D ellipse. For each trial, we set the threshold to be six times the SD of the gaze velocity ${ }^{37,39,61}$ using a median-based estimate of the SD. Small fixational saccades were defined when six or more consecutive velocity samples (i.e. a minimum of $6 \mathrm{~ms}$ ) were observed outside the ellipse.

Per standard procedure, we controlled for corrective saccades following overshoots that could have been confused with saccades. Thus, saccades were discarded when separated by less than $50 \mathrm{~ms}$ from the preceding one. We verified that the velocity and the magnitude of the saccades were correlated $(r=.78)$, thus confirming a low false alarm rate of the saccade detection algorithm ${ }^{62}$.

Visual inspection of the data revealed relatively low saccade rates across subjects. Hence, the saccade time series were smoothed using a moving average window of $100 \mathrm{~ms}$, as in ${ }^{47}$. Preliminary analyses of the results with a moving window of $50 \mathrm{~ms}$ (as in $38,59,63$ ) did not affect the data interpretation, other than reducing the signal/noise ratio. The detection of peak inhibition and rebound were restricted to the $100-300 \mathrm{~ms}$ and 300-500 ms time intervals following the offset of the warning stimulus to avoid local minima and maxima, respectively.

EEG acquisition and analysis. EEG activity was continuously collected using a Biosemi ActiveTwo 10-20 system with 64 active channels at $1024 \mathrm{~Hz}$ sampling rates and the ActiView software. The electrode offset was kept below $20 \mathrm{mV}$. The offset values were the voltage difference between each electrode and the CMS-DRL reference channels. EEG analyses were performed with MNE-Python v.0.22.0 ${ }^{64}$. 
The Autoreject algorithm ${ }^{65}$ was used to detect and repair artifacts. The motive in using this algorithm was to maximize the signal/noise ratio in adapting automatically the artifact detection parameters for each participant. It implements topographic interpolations ${ }^{66}$ to correct bad segments. One participant was removed from EEG analysis due to an excessive number of artifacts in the recording. The procedure rejected a mean average of 36 trials $(S D=8)$. A surface Laplacian filter was applied (stiffness $m=$ $4, \lambda=10^{-5}$ ) to the data resulting in reference-free current source densities (CSD) which increase the spatial resolution of the signal and reduce the signal deformation due to volume conduction ${ }^{67}$.

For the CNV analysis, the data were filtered with a $.1 \mathrm{~Hz}$ high pass filter and a $30 \mathrm{~Hz}$ low pass filter. Then, the segmentation of the trials included a time interval starting $1200 \mathrm{~ms}$ before the offset of the warning signal and ending $700 \mathrm{~ms}$ and $1300 \mathrm{~ms}$ after the offset of the warning signal for the short and long FP, respectively. We selected the electrodes presenting the peak of the CNV component (i.e. electrodes $\mathrm{C} 1, \mathrm{C2}, \mathrm{Cz}, \mathrm{CP} 1, \mathrm{CP} 2, \mathrm{CP}$, as in ${ }^{30,37}$ ) recorded over centro-parietal sites. CNV activities were then z-score normalized, using the mean average of the $200 \mathrm{~ms}$ interval before the offset of the warning signal.

For the analysis of the oscillatory activity, time-frequency representations (TFRs) were computed for each trial using a wavelet approach ${ }^{68}$. A family of Morlet wavelets (Gaussian-windowed complex sine wave) was built to perform the convolution via fast Fourier transform over each channel. The family of wavelets was parametrized to extract frequencies from $4 \mathrm{~Hz}$ to $40 \mathrm{~Hz}$. The number of cycles of wavelets was linearly-spaced, from 3 cycles for the lowest frequency to 10 cycles for the highest frequency. This precaution was used to keep a well-balanced trade-off between time and frequency resolution at each frequency. A baseline correction was applied to transform the signal amplitude into $\mathrm{dB}$ change, and then into normalized $\mathrm{z}$-score using the mean average of the $1000 \mathrm{~ms}$ interval before the offset of the warning signal. Trials were then re-segmented to remove edge artifacts, starting $100 \mathrm{~ms}$ before the offset of the warning signal and finishing at $600 \mathrm{~ms}$ and $1200 \mathrm{~ms}$ after the offset of the warning signal for the trials with short and long FP, respectively. Preliminary visualization of the oscillatory activities across the scalp revealed two main temporo-spatial clusters, namely a power increase in the theta $(4-8 \mathrm{~Hz})$ and alpha $(8-12 \mathrm{~Hz})$ bands recorded in fronto-central electrodes and a power decrease in the alpha and beta $(16-24 \mathrm{~Hz})$ bands recorded over the left motor electrodes (Fig. S4). Theta-band analysis was based on the medial fronto-central electrode FCz, where the presence of the oscillations was maximal (as in ${ }^{36}$ ).

To quantify the phase-amplitude coupling, data-driven non-linear auto-regressive models 69 were used to build comodulograms. These comodulograms reflected the influence of the phase of theta-band oscillations recorded over the medial fronto-central cortex (electrode FCz) on the amplitude of the beta-band and gamma-band $(24-40 \mathrm{~Hz})$ oscillations recorded over the left motor cortex (electrode $\mathrm{C} 3$, similar to ${ }^{36}$ ). For each participant and each cueing condition, comodulograms were computed from the current source densities of long FP trials using the entire 0-1000 ms time-interval following the offset of the warning stimulus, providing a phase and amplitude frequency resolution of $0.2 \mathrm{~Hz}$ and $1 \mathrm{~Hz}$, respectively.

RStudio (v. 0.99.489) and the rstatix (v. 0.6.0) package were used to perform two-sided repeated-measures analysis of variances (rANOVA) and planned comparisons analysis with Tukey's HSD tests corrected for multiple comparisons with the falsediscovery rate method ${ }^{70}$. For statistical analysis, EEG data were downsampled to $512 \mathrm{~Hz}$ to facilitate computations. All rANOVAs were performed with a Greenhouse-Geisser correction when within-subject factors (Cue, Foreperiod, and Block) violated the sphericity assumption. Shapiro's test was used to evaluate the normal distribution of the data. Pearson's or Spearman's correlation analyses were used depending on the normality of the distribution of the data.

\section{Declaration of interests}

The authors declare no competing interests.

\section{Acknowledgments}

The authors have no conflicts of interest to disclose. We would like to thank Profs. Hafed Ziad and Engbert Ralf for their valuable comments on the eye-tracking data. This study was funded by the EU, Horizon 2020 Framework Program, FET Proactive (VIRTUALTIMES consortium, grant agreement Id: 824128 to Anne Giersch). Also, we would like to thank Dr. Clara Alida Cutello and Raquel Neal for their valuable comments.

\section{Author Contributions}

Conceptualization, Methodology and Writing - Original Draft, F.R.F. and A.G.; Software, Investigation and Visualization F.R.F.; Writing - Review \& Editing, F.R.F., M.C., A.B. \& A.G.; Funding Acquisition, Resources and Supervision, A.G. All authors approved the final version of the manuscript for submission.

\section{References}

1. Petry, N. M. \& O'Brien, C. P. Internet gaming disorder and the DSM-5. Addiction 108, 1186-1187 (2013).

2. King, D. L. \& Delfabbro, P. H. The cognitive psychology of Internet gaming disorder. Clin. Psychol. Rev. 34, 298-308 (2014).

3. American Psychiatric Association. Diagnostic and Statistical Manual of Mental Disorders. (American Psychiatric Association, 2013). doi:10.1176/appi.books.9780890425596

4. Bavelier, D. et al. Brains on video games. Nat. Rev. Neurosci. 12, 763-768 (2011). 
$428 \quad 5 . \quad$ Bavelier, D., Green, C. S., Pouget, A. \& Schrater, P. Brain Plasticity Through the Life Span: Learning to Learn and Action Video Games. Annu. Rev. Neurosci. 391-416 (2012). doi:10.1146/060909-152832

6. Powers, K. L., Brooks, P. J., Aldrich, N. J., Palladino, M. A. \& Alfieri, L. Effects of video-game play on information processing: A meta-analytic investigation. Psychon. Bull. Rev. 20, 1055-1079 (2013).

7. Bediou, B. et al. Meta-analysis of action video game impact on perceptual, attentional, and cognitive skills. Psychol. Bull. 144, 77-110 (2018).

8. Vallesi, A., Shallice, T. \& Walsh, V. Role of the Prefrontal Cortex in the Foreperiod Effect: TMS Evidence for Dual Mechanisms in Temporal Preparation. 466-474 (2007). doi:10.1093/cercor/bhj163

9. Coull, J. T. \& Nobre, A. C. Dissociating explicit timing from temporal expectation with fMRI. Curr. Opin. Neurobiol. 18, 137-144 (2008).

10. Coull, J. T., Cheng, R. \& Meck, W. H. Neuroanatomical and Neurochemical Substrates of Timing. Neuropsychopharmacology 3-25 (2011). doi:10.1038/npp.2010.113

11. Balasubramaniam, R. et al. Neural Encoding and Representation of Time for Sensorimotor Control and Learning. J. Neurosci. 41, 866-872 (2021).

12. Wright, W. G. Using virtual reality to augment perception, enhance sensorimotor adaptation, and change our minds. 8, 1-6 (2014).

13. Van Der Mijn, R. \& Van Rijn, H. Attention Does Not Affect the Speed of Subjective Time, but Whether Temporal Information Guides Performance : A Large-Scale Study of Intrinsically Motivated Timers in a Real-Time Strategy Game. Cogn. Sci. 45, (2021).

14. West, G. L., Stevens, S. A., Pun, C. \& Pratt, J. Visuospatial experience modulates attentional capture: Evidence from action video game players. J. Vis. 8, 1-9 (2008).

15. Krishnan, L., Kang, A., Sperling, G. \& Srinivasan, R. Neural strategies for selective attention distinguish fast-action video game players. Brain Topogr. 26, 83-97 (2013).

16. Anguera, J. A. et al. Video game training enhances cognitive control in older adults. Nature 501, 97-101 (2013).

17. Bertoni, S. et al. Action video games enhance attentional control and phonological decoding in children with developmental dyslexia. Brain Sci. 11, 1-18 (2021).

18. Bavelier, D., Achtman, R. L., Mani, M. \& Föcker, J. Neural bases of selective attention in action video game players. Vision Res. 61, 132-143 (2012).

19. Mishra, J., Zinni, M., Bavelier, D. \& Hillyard, S. A. Neural Basis of Superior Performance of Action Videogame Players in an Attention-Demanding Task. J. Neurosci. 31, 992-998 (2011).

20. Dye, M. W. G., Green, C. S. \& Bavelier, D. The development of attention skills in action video game players. Neuropsychologia 47, 1780-1789 (2009).

21. Föcker, J., Cole, D., Beer, A. L. \& Bavelier, D. Neural bases of enhanced attentional control: Lessons from action video game players. Brain Behav. 8, 1-18 (2018).

22. Shams, T. A. et al. The Effects of Video Games on Cognition and Brain Structure: Potential Implications for Neuropsychiatric Disorders. Curr. Psychiatry Rep. 17, (2015).

23. Kühn, S., Berna, F., Lüdtke, T., Gallinat, J. \& Moritz, S. Fighting depression: Action video game play may reduce rumination and increase subjective and objective cognition in depressed patients. Front. Psychol. 9, 1-10 (2018).

24. Correa, Á. \& Nobre, A. C. Neural Modulation by Regularity and Passage of Time. J. Neurophysiol. 100, 1649-1655 (2008).

25. Correa, Á., Lupiáñez, J. \& Tudela, P. The attentional mechanism of temporal orienting: Determinants and attributes. Exp. Brain Res. 169, 58-68 (2006).

26. Vallesi, A., Lozano, V. N. \& Correa, Á. Dissociating temporal preparation processes as a function of the inter-trial interval duration. Cognition 127, 22-30 (2013).

27. Niemi, P. \& Näätänen, R. The foreperiod and simple reaction time. Psychol. Bull. 89, 133-162 (1981).

28. Mattes, S. \& Rolf, U. Response force is sensitive to the temporal uncertainty of response stimuli. Percept. Psychophys. 59, 1089-1097 (1997).

29. Cravo, A. M., Rohenkohl, G., Santos, K. M. \& Nobre, A. C. Temporal Anticipation Based on Memory. J. Cogn. Neurosci. 29, 2081-2089 (2017).

30. Praamstra, P. Neurophysiology of Implicit Timing in Serial Choice Reaction-Time Performance. J. Neurosci. 26, 54485455 (2006).

31. Pfeuty, M., Ragot, R. \& Pouthas, V. Relationship between CNV and timing of an upcoming event. Neurosci. Lett. 382, 106-111 (2005).

32. Duma, G. M., Granziol, U. \& Mento, G. Should I stay or should I go? How local-global implicit temporal expectancy shapes proactive motor control: An hdEEG study. Neuroimage 220, 117071 (2020).

33. Mento, G. The role of the P3 and CNV components in voluntary and automatic temporal orienting: A high spatialresolution ERP study. Neuropsychologia 107, 31-40 (2017).

34. Mento, G., Tarantino, V., Vallesi, A. \& Bisiacchi, P. S. Spatiotemporal Neurodynamics Underlying Internally and Externally Driven Temporal Prediction: A High Spatial Resolution ERP Study. J. Cogn. Neurosci. 27, 425-439 (2015). 
35. van Rijn, H., Kononowicz, T. W., Meck, W. H., Ng, K. K. \& Penney, T. B. Contingent negative variation and its relation to time estimation: a theoretical evaluation. Front. Integr. Neurosci. 5, 91 (2011).

36. Cravo, A. M., Rohenkohl, G., Wyart, V. \& Nobre, A. C. Endogenous modulation of low frequency oscillations by temporal expectations. J. Neurophysiol. 106, 2964-2972 (2011).

37. Amit, R., Abeles, D., Carrasco, M. \& Yuval-Greenberg, S. Oculomotor inhibition reflects temporal expectations. Neuroimage 184, 279-292 (2019).

38. Tal-Perry, N. \& Yuval-Greenberg, S. Pre-target oculomotor inhibition reflects temporal orienting rather than certainty. Sci. Rep. 10, 1-9 (2020).

39. Abeles, D., Amit, R., Tal-Perry, N., Carrasco, M. \& Yuval-Greenberg, S. Oculomotor inhibition precedes temporally expected auditory targets. Nat. Commun. 11, 1-12 (2020).

40. Badde, S., Myers, C. F., Yuval-Greenberg, S. \& Carrasco, M. Oculomotor freezing reflects tactile temporal expectation and aids tactile perception. Nat. Commun. 11, 1-9 (2020).

41. Gentile, D. A. et al. Internet Gaming Disorder in Children and Adolescents. Pediatrics 140, S81-S85 (2017).

42. Zhang, R. Y. et al. Action video game play facilitates "learning to learn". Commun. Biol. 4, (2021).

43. White, A. L. \& Rolfs, M. Oculomotor inhibition covaries with conscious detection. J. Neurophysiol. 116, 1507-1521 (2016).

44. Engbert, R. \& Kliegl, R. Microsaccades uncover the orientation of covert attention. Vision Res. 43, 1035-1045 (2003).

45. Hafed, Z. M. \& Ignashchenkova, A. On the dissociation between microsaccade rate and direction after peripheral cues: Microsaccadic inhibition revisited. J. Neurosci. 33, 16220-16235 (2013).

46. Hafed, Z. M., Yoshida, M., Tian, X., Buonocore, A. \& Malevich, T. Dissociable Cortical and Subcortical Mechanisms for Mediating the Influences of Visual Cues on Microsaccadic Eye Movements. Front. Neural Circuits 15, 1-18 (2021).

47. Betta, E. \& Turatto, M. Are you ready? I can tell by looking at your microsaccades. Neuroreport 17, 1001-1004 (2006).

48. Glaholt, M. G. \& Reingold, E. M. Perceptual enhancement as a result of a top-down attentional influence in a scene viewing task: Evidence from saccadic inhibition. Q. J. Exp. Psychol. 71, 56-63 (2018).

49. Mento, G., Astle, D. E. \& Scerif, G. Cross-frequency Phase-Amplitude Coupling as a Mechanism for Temporal Orienting of Attention in Childhood. J. Cogn. Neurosci. 30, 594-602 (2018).

50. Grabot, L. et al. The strength of alpha-beta oscillatory coupling predicts motor timing precision. J. Neurosci. 39, 32773291 (2019).

51. Loughnane, G. M., Newman, D. P., Tamang, S., Kelly, S. P. \& O'Connell, R. G. Antagonistic interactions between microsaccades and evidence accumulation processes during decision formation. J. Neurosci. 38, 2163-2176 (2018).

52. Canolty, R. T. \& Knight, R. T. The functional role of cross-frequency coupling. Trends Cogn. Sci. 14, 506-515 (2010).

53. Coull, J. T., Cotti, J. \& Vidal, F. Differential roles for parietal and frontal cortices in fi xed versus evolving temporal expectations : Dissociating prior from posterior temporal probabilities with fMRI. Neuroimage 141, 40-51 (2016).

54. Bejjanki, V. R. et al. Action video game play facilitates the development of better perceptual templates. Proc. Natl. Acad. Sci. U. S. A. 111, 16961-16966 (2014).

55. Green, C. S. \& Bavelier, D. Learning, Attentional Control, and Action Video Games. Curr. Biol. 22, R197-R206 (2012).

56. Franceschini, S. et al. Action video games make dyslexic children read better. Curr. Biol. 23, 462-466 (2013).

57. Bach, M. The Freiburg Visual Acuity Test - Automatic Measurement of Visual Acuity. Optom. Vis. Sci. Vol. 73, p 49-53 (1996).

58. Edman, G., Schalling, D. \& Levander, S. E. Impulsivity and speed and errors in a reaction time task: A contribution to the construct validity of the concept of impulsivity. Acta Psychol. (Amst). 53, 1-8 (1983).

59. Dankner, Y., Shalev, L., Carrasco, M. \& Yuval-Greenberg, S. Prestimulus Inhibition of Saccades in Adults With and Without Attention-Deficit/Hyperactivity Disorder as an Index of Temporal Expectations. Psychol. Sci. 28, 835-850 (2017).

60. Engbert, R. Microsaccades: a microcosm for research on oculomotor control, attention, and visual perception. Prog. Brain Res. 154, 177-192 (2006).

61. Rolfs, M., Kliegl, R. \& Engbert, R. Toward a model of microsaccade generation: The case of microsaccadic inhibition. J. Vis. 8, 5-5 (2008).

62. Zuber, B. L., Start, L. \& Cook, G. Velocity-Amplitude Relationship. Science (80-. ). 150, 1459-1460 (1965).

63. Denison, R. N., Yuval-Greenberg, S. \& Carrasco, M. Directing Voluntary Temporal Attention Increases Fixational Stability. J. Neurosci. 39, 353-363 (2019).

64. Gramfort, A. et al. MNE software for processing MEG and EEG data. Neuroimage 86, 446-460 (2014).

65. Jas, M., Engemann, D. A., Bekhti, Y., Raimondo, F. \& Gramfort, A. Autoreject: Automated artifact rejection for MEG and EEG data. Neuroimage 159, 417-429 (2017).

66. Perrin, F., Pernier, J., Bertrand, O. \& Echallier, J. F. Spherical splines for scalp potential and current density mapping. Electroencephalogr. Clin. Neurophysiol. 72, 184-187 (1989).

67. Kayser, J. \& Tenke, C. E. On the benefits of using surface Laplacian (current source density) methodology in electrophysiology. Int. J. Psychophysiol. 97, 171-173 (2015).

68. Tallon-Baudry, C. \& Bertrand, O. Oscillatory gamma activity in humans and its role in object representation. Trends Cogn. Sci. 3, 151-162 (1999). 
Neurocognitive evidence of enhanced implicit temporal processing in video game players

544

545

546

547

548

549

69. Dupré la Tour, T. et al. Non-linear auto-regressive models for cross-frequency coupling in neural time series. PLOS Comput. Biol. 13, e1005893 (2017).

70. Benjamini, Y. \& Hochberg, Y. Controlling the False Discovery Rate: A Practical and Powerful Approach to Multiple Testing. J. R. Stat. Soc. Ser. B 57, 289-300 (1995). 
STAR Methods

\begin{tabular}{|c|c|c|}
\hline REAGENT or RESOURCE & SOURCE & IDENTIFIER \\
\hline \multicolumn{3}{|c|}{ Deposited data } \\
\hline Processed data (behavior, EEG and eye-tracking) & $\underline{\text { https://osf.io/54pj7/ }}$ & \\
\hline \multicolumn{3}{|c|}{ Software and Algorithms } \\
\hline$R(v .3 .6 .1)$ & https://www.r-project.org & RRID:SCR_000432 \\
\hline MNE-Python (v.0.22.0) & https://mne.tools/stable/index.html & RRID:SCR_005972 \\
\hline \multicolumn{3}{|l|}{ Rstatix-package (v. 0.6.0) } \\
\hline Unity (v. 2019.3.9f1) & Unity technologies & \\
\hline ActiView & Biosemi & \\
\hline \multicolumn{3}{|c|}{ Other } \\
\hline $\mathrm{R}$ code for data analysis (behavior, EEG and eye-tracking) & https://osf.io/54pj7/ & \\
\hline
\end{tabular}

\section{RESOURCE AVAILABILITY}

\section{Lead contact}

Further information and requests for resources should be directed to and will be fulfilled by the Lead Contact, François R. Foerster (francois.foerster@gmail.com).

\section{Data availability}

Data and codes are available in the open science framework (OSF), accessible at https://osf.io/54pj7/.

\section{EXPERIMENTAL MODEL AND SUBJECT DETAILS}

Participants. The VGP group concerned 23 participants ( 4 females, 2 left-handed, age Mean $=25.2 ; S D=5.7$ ). The criterion to be considered a VGP was a minimum of 5 hours per week of action video game practice for the previous 12 months, as reported in previous studies 18,21,54. The games mainly included first-person shooters (e.g. Call of Duty series, Apex Legends, Overwatch, Counter Striker series), multiplayer online battle arena (e.g. Leagues of Legends, Heroes of the Storm), and real-time strategy (Starcraft II) which involve important visual and timing expectations, as well as high-speed visual processing and motor responses to optimize game performance. The NVGP group concerned 23 participants ( 7 females, 5 left-handed, age Mean = 26.8; SD = 4.6). The criterion to be included in the NVGP group was little or no action video game practice for a minimum of one year, although no extensive practice ever $(\mathrm{N}=16)$ was highly favored. All subjects had normal or corrected-to-normal visual acuity, as checked with the Freiburg Visual Acuity Test ${ }^{57}$. One VGP has been removed from the EEG analysis due to excessive noise in the recorded signal. All subjects were given a compensation of $45 €$ for their participation. The study has been approved by the local ethics committee of the University of Strasbourg (i.e. Comité d'Éthique de Recherche).

\section{METHOD DETAILS}

Experimental Protocol. The experiment used the Unity software (Unity technologies, v. 2019.3.9f1) to create the virtual environment. The HTC Vive Eye Pro (HTC Corp.) headset and controllers were used to immerse the participants in VR. Participants wore both the EEG and VR headsets while sitting on a chair. The use of VR has several advantages. Firstly, VR allows a better trade-off between fully-controlled experimental settings and ecological experience (e.g. 3D visual percepts) in comparison with 2D screen setups. Secondly, it increases the engagement of the participant to the task at hand. Thirdly, the embedded eyetracking system to the VR headset allows researchers to easily track and record the gaze in the 3D space. Eye-tracking was used to trigger visual stimuli. Participants were instructed to fixate the warning signal without moving their eyes, and it was only after a time interval free of saccades and eye-blinks that the warning signal was switched off. This procedure improves the data quality of EEG recordings.

Each participant was immersed in a virtual room, facing four 3D robots, each with a light whose color and onset were manipulated (Figure 1A). The experiment was composed of two intertwined tasks: a variable foreperiod task and an asynchrony detection task. The asynchrony detection task consisted of discriminating whether the lights of two robots appeared synchronously or asynchronously (using delays of $11 \mathrm{~ms}, 33 \mathrm{~ms}$, or $66 \mathrm{~ms}$ ). Participants performed four blocks of trials for each task. The participant switched tasks every block to reduce boredom. At the end of each experimental block, a break was proposed to the participant to remove the VR headset. Given the research questions investigated in this article, only data collected from the variable foreperiod task are presented. 
Variable foreperiod task and stimuli. In case the button was pressed before the go-signal, a warning sound was delivered to the participant signaling the incorrect response. The procedure consisted of 4 blocks of 120 trials, with each block comprising 60 trials with short (S) FP and 60 trials with (L) long FP. The procedure excluded the possibility of having three same foreperiods in a row (i.e. SSS or LLL). The light of the robots was presented at a distance of 4 meters from the participant. These lights were located at $8^{\circ}$ and $24^{\circ}$ of visual angle from the center of the scene. The presentation of the temporal $(\mathrm{T})$ and neutral (N) cue conditions was alternated, taking the form of NTNT $(\mathrm{N}=24)$ or TNTN $(\mathrm{N}=22)$. At the beginning of each block, the two robots used in the condition were relocated to the center of the scene and the two other robots were relocated on the sides (randomly on the right and left). At the beginning of each trial, a colored (during temporal cue blocks) or uncolored (during neutral cue blocks) robot was randomly selected to include the warning signal and the target light. The matching of the robot's color with the FP was randomly assigned for each participant: blue for the short FP and turquoise for the long FP, or the reverse.

\section{QUANTIFICATION AND STATISTICAL ANALYSIS}

Behavioral analyses. On the one hand, pressing the button before the onset of the target (i.e. anticipated responses) reveal the impulsivity 58 in the two groups. On the other hand, pressing the button after the onset of the target was used to compute the two indexes (i.e. benefits from the passage of time and temporal attention cue).

Eye-tracking acquisition and analyses. The binocular gaze position was monitored using the eye-tracking system (Tobii Ltd.) embedded in the VR headset at a sampling rate of $90 \mathrm{~Hz}$ and an estimated spatial accuracy between $0.5^{\circ}$ to $1.1^{\circ}$. The particularity of such a system is that 1 ) it tracks the gaze position independently of head movements, 2 ) it provides estimations of the gaze location in the 3D space rather than on-screen 2D space, 3) the calibration-free data recording for saccades analysis renders the measure non-intrusive. Here we analyzed the likelihood of small fixational saccades during the foreperiods, as previously investigated $37,39,59$. Saccades of all sizes were included, but due to the task requirements to fixate the stimulus area, most saccades were small ( $1.4^{\circ}$ of visual angle on average).

First, the onsets of blinks were identified with the HTC SRanipal SDK, detecting blinks via individual eye openness. Because blinks were particularly rare events given to non-blinking requirements to trigger the offset of the warning signal, trials containing at least one blink occurring during the time-window of interest (i.e. -200 to $600 \mathrm{~ms}$ in trials with short FP; -200 to 1200 $\mathrm{ms}$ in trials with long FP) were discarded ( $5.3 \%$ of total trials). Trials with anticipated responses were also discarded ( $1.85 \%$ of the data). Second, raw data (i.e. the 3D gaze position over time) of each trial were interpolated with a spline method to increase the temporal precision followed by the calculations of the derivations of the speed of vertical and horizontal movements.

Saccades were detected using a modification of a published algorithm ${ }^{60}$ based on gaze's velocities. A threshold criterion for saccades detection was determined in the 2D velocity space based on the horizontal and the vertical velocities of gaze movement. This 2D space represented a plane surface located at the stimulus area (i.e. the warning signal and target). This threshold was represented by a 2D ellipse. For each trial, we set the threshold to be six times the SD of the gaze velocity ${ }^{37,39,61}$ using a median-based estimate of the SD. Small fixational saccades were defined when six or more consecutive velocity samples (i.e. a minimum of $6 \mathrm{~ms}$ ) were observed outside the ellipse.

Per standard procedure, we controlled for corrective saccades following overshoots that could have been confused with saccades. Thus, saccades were discarded when separated by less than $50 \mathrm{~ms}$ from the preceding one. We verified that the velocity and the magnitude of the saccades were correlated $(r=.78)$, thus confirming a low false alarm rate of the saccade detection algorithm ${ }^{62}$.

Visual inspection of the data revealed relatively low saccade rates across subjects. Hence, the saccade time series were smoothed using a moving average window of $100 \mathrm{~ms}$, as in ${ }^{47}$. Preliminary analyses of the results with a moving window of $50 \mathrm{~ms}$ (as in ${ }^{38,59,63}$ ) did not affect the data interpretation, other than reducing the signal/noise ratio. The detection of peak inhibition and rebound were restricted to the $100-300 \mathrm{~ms}$ and 300-500 ms time intervals following the offset of the warning stimulus to avoid local minima and maxima, respectively.

EEG acquisition and analysis. EEG activity was continuously collected using a Biosemi ActiveTwo 10-20 system with 64 active channels at $1024 \mathrm{~Hz}$ sampling rates and the ActiView software. The electrode offset was kept below $20 \mathrm{mV}$. The offset values were the voltage difference between each electrode and the CMS-DRL reference channels. EEG analyses were performed with MNE-Python v.0.22.0 ${ }^{64}$.

The Autoreject algorithm ${ }^{65}$ was used to detect and repair artifacts. The motive in using this algorithm was to maximize the signal/noise ratio in adapting automatically the artifact detection parameters for each participant. It implements topographic interpolations ${ }^{66}$ to correct bad segments. One participant was removed from EEG analysis due to an excessive number of artifacts in the recording. The procedure rejected a mean average of 36 trials $(S D=8)$. A surface Laplacian filter was applied (stiffness $m=$ $4, \lambda=10^{-5}$ ) to the data resulting in reference-free current source densities (CSD) which increase the spatial resolution of the signal and reduce the signal deformation due to volume conduction ${ }^{67}$.

For the CNV analysis, the data were filtered with a $.1 \mathrm{~Hz}$ high pass filter and a $30 \mathrm{~Hz}$ low pass filter. Then, the segmentation of the trials included a time interval starting $1200 \mathrm{~ms}$ before the offset of the warning signal and ending $700 \mathrm{~ms}$ and $1300 \mathrm{~ms}$ after the offset of the warning signal for the short and long FP, respectively. We selected the electrodes presenting 
the peak of the CNV component (i.e. electrodes $\mathrm{C} 1, \mathrm{C} 2, \mathrm{Cz}, \mathrm{CP} 1, \mathrm{CP} 2, \mathrm{CPz}$, as in ${ }^{30,37}$ ) recorded over centro-parietal sites. CNV activities were then $z$-score normalized, using the mean average of the $200 \mathrm{~ms}$ interval before the offset of the warning signal.

For the analysis of the oscillatory activity, time-frequency representations (TFRs) were computed for each trial using a wavelet approach ${ }^{68}$. A family of Morlet wavelets (Gaussian-windowed complex sine wave) was built to perform the convolution via fast Fourier transform over each channel. The family of wavelets was parametrized to extract frequencies from $4 \mathrm{~Hz}$ to $40 \mathrm{~Hz}$. The number of cycles of wavelets was linearly-spaced, from 3 cycles for the lowest frequency to 10 cycles for the highest frequency. This precaution was used to keep a well-balanced trade-off between time and frequency resolution at each frequency. A baseline correction was applied to transform the signal amplitude into $\mathrm{dB}$ change, and then into normalized z-score using the mean average of the $1000 \mathrm{~ms}$ interval before the offset of the warning signal. Trials were then re-segmented to remove edge artifacts, starting $100 \mathrm{~ms}$ before the offset of the warning signal and finishing at $600 \mathrm{~ms}$ and $1200 \mathrm{~ms}$ after the offset of the warning signal for the trials with short and long FP, respectively. Preliminary visualization of the oscillatory activities across the scalp revealed two main temporo-spatial clusters, namely a power increase in the theta $(4-8 \mathrm{~Hz})$ and alpha $(8-12 \mathrm{~Hz})$ bands recorded in fronto-central electrodes and a power decrease in the alpha and beta $(16-24 \mathrm{~Hz})$ bands recorded over the left motor electrodes (Fig. S4). Theta-band analysis was based on the medial fronto-central electrode FCz, where the presence of the oscillations was maximal (as in ${ }^{36}$ ).

To quantify the phase-amplitude coupling, data-driven non-linear auto-regressive models 69 were used to build comodulograms. These comodulograms reflected the influence of the phase of theta-band oscillations recorded over the medial fronto-central cortex (electrode $\mathrm{FCz}$ ) on the amplitude of the beta-band and gamma-band $(24-40 \mathrm{~Hz})$ oscillations recorded over the left motor cortex (electrode $\mathrm{C} 3$, similar to ${ }^{36}$ ). For each participant and each cueing condition, comodulograms were computed from the current source densities of long FP trials using the entire 0-1000 ms time-interval following the offset of the warning stimulus, providing a phase and amplitude frequency resolution of $0.2 \mathrm{~Hz}$ and $1 \mathrm{~Hz}$, respectively.

\section{Significance testing}

$R$ (v. 3.6.1) sofware and the rstatix (v. 0.6.0) package were used to perform two-sided repeated-measures analysis of variances (rANOVA) and planned comparisons analysis with Tukey's HSD tests corrected for multiple comparisons with the falsediscovery rate method ${ }^{70}$. For statistical analysis, EEG data were downsampled to $512 \mathrm{~Hz}$ to facilitate computations. All rANOVAs were performed with a Greenhouse-Geisser correction when within-subject factors (Cue, Foreperiod, and Block) violated the sphericity assumption. Shapiro's test was used to evaluate the normal distribution of the data. Pearson's or Spearman's correlation analyses were used depending on the normality of the distribution of the data. 


\section{Supplementary Files}

This is a list of supplementary files associated with this preprint. Click to download.

- SupplMatNatCommBiol.pdf 